\title{
Serum HE4 concentration differentiates malignant ovarian tumours from ovarian endometriotic cysts
}

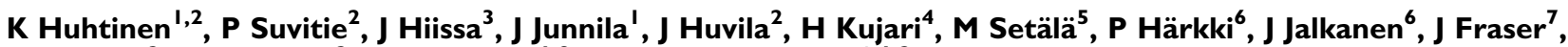 \\ J Mäkinen ${ }^{2}$, A Auranen ${ }^{2}$, M Poutanen ${ }^{1,8}$ and A Perheentupa ${ }^{*, 1,2}$
}

'Department of Physiology, Institute of Biomedicine, University of Turku, Fl-20520 Turku, Finland; '2Department of Obstetrics and Gynaecology, Turku University Hospital, Fl-20520 Turku, Finland; ${ }^{3}$ Department of Mathematics, University of Turku, Fl-200 14 Turku, Finland; ${ }^{4}$ Department of Pathology, Turku University Hospital, Fl-20520 Turku, Finland; ${ }^{5}$ Department of Obstetrics and Gynaecology, Päijät-Häme Central Hospital, Fl- 5850 Lahti, Finland;

${ }^{6}$ Department of Obstetrics and Gynaecology, Helsinki University Hospital, Fl-00029 Helsinki, Finland; ' Department of Obstetrics and Gynaecology, North Karelia Central Hospital, Fl-802 10 Joensuu, Finland; ${ }^{8}$ Turku Center for Disease Modeling, University of Turku, Fl-20520 Turku, Finland

\begin{abstract}
Human epididymal secretory protein E4 (HE4, also known as WAP four-disulphide core domain protein 2) is a new promising biomarker for ovarian cancer but its specificity against ovarian endometriotic cysts is only superficially known. We, thus, analysed serum HE4 concentrations together with a tumour marker CAI 25 in serum samples of women diagnosed with various types of endometriosis, endometrial cancer or ovarian cancer, and in samples from healthy controls. The mean serum concentration of HE4 was significantly higher in serum samples of patients with both endometrial $(99.2 \mathrm{pM}, \mathrm{P}<0.00 \mathrm{I}$ ) and ovarian (I I $25.4 \mathrm{pM}, \mathrm{P}<0.00 \mathrm{I}$ ) cancer but not with ovarian endometriomas $(46.0 \mathrm{pM})$ or other types of endometriosis $(45.5 \mathrm{pM})$ as compared with healthy controls (40.5 pM). The serum CAI 25 concentrations were elevated in patients with ovarian cancer, advanced endometriosis with peritoneal or deep lesions, or ovarian endometriomas, but not in the patients with endometrial cancer. The microarray results revealed that the mRNA expression of the genes encoding HE4 and CAI 25 reflected the serum protein concentrations. Taken together, measuring both HE4 and CAI 25 serum concentrations increases the accuracy of ovarian cancer diagnosis and provides valuable information to discriminate ovarian tumours from ovarian endometriotic cysts.
\end{abstract}

British Journal of Cancer (2009) I 00, I315-1319. doi: 10.1038/sj.bjc.66050 I I www.bjcancer.com

Published online 3I March 2009

(c) 2009 Cancer Research UK

Keywords: HE4; CA 125; endometriosis; ovarian cancer

Endometriosis is one of the most common benign gynaecological conditions, it may affect up to $10 \%$ of women in reproductive age. The typical symptoms caused by endometriosis are pain and subfertility. Endometriosis is characterised by the appearance of endometrial glands and stroma in ectopic locations outside the uterine cavity. The lesions are typically located on the peritoneum, in the ovaries or infiltered into the organs within the abdominal cavity (Giudice and Kao, 2004). There are several theories for the aetiology of endometriosis and it has been suggested that the peritoneal lesions, deep rectovaginal lesions, and ovarian endometriotic cysts called endometriomas would be of different origin (Nisolle and Donnez, 1997).

Although transvaginal ultrasound examination is of value, particularly in women with ovarian endometriomas, the diagnosis of endometriosis typically requires laparoscopic verification. The ultrasound is not a useful tool in the diagnostics of peritoneal implants and adhesions or in staging of the disease. In addition, neoplastic ovarian cysts can resemble endometriomas in ultrasound and, therefore, need to be carefully considered in the

*Correspondence: Dr A Perheentupa; E-mail: antti.perheentupa@utu.fi Received 27 January 2009; revised 2 March 2009; accepted 5 March 2009; published online 31 March 2009 differential diagnostics. Furthermore, endometriosis is shown to increase the risk of certain subtypes of ovarian cancer, such as endometrioid and clear-cell carcinomas (Nagle et al, 2008). There are data indicating that $40 \%$ of endometrioid ovarian carcinomas and $50 \%$ of clear-cell ovarian carcinomas are associated with endometriosis (Sato et al, 2000). Both endometrioid and clear-cell carcinomas are thought to arise, at least partly, from endometriosis. Similar pathophysiological mechanisms may be involved in the progression of endometriosis as well as in its transformation into ovarian neoplasia (Ness, 2003).

Currently, CA125 antigen is the most commonly used biochemical marker in ovarian cancer diagnostics. However, it is associated with a high false-positive rate among women with benign gynaecological conditions such as endometriosis (Markman, 1997). Furthermore, CA125 has very low sensitivity in identifying patients with early-stage ovarian cancer (Terry et al, 2004). Thus, to improve the specificity and sensitivity of ovarian cancer diagnosis, the use of novel biomarkers such as HE4 (human epididymal secretory protein E4; WAP four-disulphide core domain protein 2, WFDC2) alone or in combination with CA125 has been intensively studied (Hellström et al, 2003; Havrilesky et al, 2008; Moore et al, 2008a).

In addition to diagnosis of ovarian cancer, CA125 can be used to evaluate the efficacy of endometriosis therapy as well as the recurrence of endometriosis. However, the lack of sensitivity and 
specificity of the biomarker has significantly hampered its use as a diagnostic test (Mol et al, 1998; Kitawaki et al, 2005). On the other hand, a panel of markers including CA125 has been suggested to predict the presence of endometriosis in a subset of patients (Seeber et al, 2008).

Human epididymal secretory protein E4 is a new serological biomarker for diagnosis of ovarian cancer. As compared with the markers used previously, it possesses increased sensitivity for detecting ovarian cancer, especially the stage I disease (Havrilesky et al, 2008; Moore et al, 2008a), whereas the HE4 encoding gene WFDC2 is expressed particularly in serous and endometrioid ovarian cancer (Drapkin et al, 2005; Galgano et al, 2006). However, it is shown to be expressed also in some other types of tumours, for example lung adenocarcinoma (Galgano et al, 2006). Its expression is also apparent in normal endometrial glands and endometrial cancer (Drapkin et al, 2005; Galgano et al, 2006), but the expression in endometriotic lesions including ovarian endometriomas is not known.

In the present study, we measured serum concentrations of HE4 and CA125 in 129 patients with endometriosis of whom 69 had ovarian endometriomas. The data were compared with results obtained in 16 patients with endometrial cancer, 14 with ovarian cancer, and 66 healthy controls. The mRNA expression of genes encoding HE4 and CA125, namely WFDC2 and MUC16, respectively, was also analysed in the tissue specimens of various types of endometriosis, ovarian cancer, endometrial cancer, and normal endometrium.

\section{PATIENTS AND METHODS}

\section{Patients}

The patients were enrolled into the study in two Central Hospitals and two University Central Hospitals in Finland between October 2005 and November 2007. A written informed consent was required from all patients before sampling, and the study protocol was approved by the joint ethics committee of Turku University and Turku University Central Hospital, Turku, Finland.

The serum samples of women diagnosed with endometriosis (Endo, $n=129$ ), ovarian cancer (OvCa, $n=14$ ), or endometrial cancer (EmCa, $n=16)$ were included into the study, together with 66 samples collected from healthy controls (Ctrl). The serum samples of patients with ovarian endometrioma (OvEndo, $n=69$, ASRM stage 3-4) were evaluated as a separate group in the analysis. The diseases were diagnosed per operatively in laparoscopy or laparotomy and confirmed by histopathological evaluation. Description of patients included in the study is presented in Table 1. Patients with endometriosis were classified to stage 1-4 according to the revised American Society for Reproductive Medicine (ASRM) criteria (1997). The patients with ovarian and endometrial cancer were staged according to the FIGO guidelines (Benedet et al, 2000). The 14 ovarian carcinomas included 7 serous, 3 mucinous, 2 clear-cell, 1 endometrioid, and 1 small-cell carcinomas. Four of the ovarian cancers were local stage I cancers and the remaining 10 were of advanced stage II-IV. All endometrial carcinomas were endometrioid adenocarcinomas. In 14 patients the cancer was limited to the uterus (stage I-II), whereas in 2 cases metastatic pelvic lymph nodes were present (stage III). Control subjects $(n=66)$ were verified to be free from endometriosis or ovarian cancer by laparoscopy during the tubal sterilisation, and the possibility of endometrial cancer was excluded by endometrial biopsy. The mean age of patients with endometriosis, ovarian cancer, endometrial cancer, and healthy controls was $31.8,63.8,60.5$, and 38.5 years, respectively.

The gene expression of WFDC2 and MUC16, which encode the HE4 and CA125 proteins, respectively, was evaluated in 149 nonovarian endometriotic lesions, 28 ovarian endometriotic cysts, 15 ovarian and 14 endometrium carcinomas, 64 individual
Table I Description of patients included in the analysis of serum HE4 and CAI25

\begin{tabular}{|c|c|c|c|c|c|}
\hline \multirow[b]{2}{*}{ Diagnosis } & \multirow[b]{2}{*}{$n$} & \multicolumn{4}{|c|}{ Classification (ASRM)/stage (FIGO) } \\
\hline & & I/I & $2 / I I$ & $3 / I I I$ & $4 / \mathrm{VI}$ \\
\hline Healthy & 66 & & & & \\
\hline $\begin{array}{l}\text { Endometriosis all } \\
\text { Ovarian endometrioma }\end{array}$ & $\begin{array}{r}129 \\
69\end{array}$ & 16 & 17 & 33 & $\begin{array}{l}63 \\
45\end{array}$ \\
\hline Ovarian endometrioma & 69 & - & - & 24 & 45 \\
\hline Endometrial cancer & 16 & 13 & 1 & 2 & - \\
\hline Ovarian cancer & 14 & 4 & 1 & 7 & 2 \\
\hline Serous & 7 & - & - & 6 & 1 \\
\hline Mucinous & 3 & 3 & - & - & - \\
\hline Clear cell & 2 & - & 1 & 1 & - \\
\hline Endometrioid & I & 1 & - & - & - \\
\hline Small cell & । & - & - & - & 1 \\
\hline Total & 225 & & & & \\
\hline
\end{tabular}

endometrium samples from patients with endometriosis, and 41 endometrium samples from healthy controls. Thirteen of the ovarian carcinomas were serous, one was endometrioid and one undifferentiated. All endometrial carcinomas were endometrioid adenocarcinomas. The tissue samples were collected during laparoscopic or open surgery. Endometrial biopsies were collected using a sterile Pipelle sampler (Pipelle de Cornier; Laboratoire CCD, Paris, France). All tissue samples were snap-frozen and stored in liquid nitrogen.

\section{Serum HE4 and CA125 analysis}

Serum samples were collected just before surgery into nonheparinised tubes and centrifuged for $15 \mathrm{~min}$ at 3000 r.p.m. $(800 \mathrm{~g})$ after keeping $30 \mathrm{~min}$ at room temperature. The serum was stored at -20 or $-80^{\circ} \mathrm{C}$. Human epididymal secretory protein E4 and CA125 concentrations were analysed in serum samples by ELISA analysis (Fujirebio Diagnostics Inc., Malvern, PA, USA) according to manufacturer's instructions.

\section{Expression analysis}

The gene expression levels of WFDC2 and MUC16 were studied as part of our whole-genome microarray analysis. The total RNA was isolated with Trizol reagent (Invitrogen, Carlsbad, CA, USA), further purified with RNeasy columns (Qiagen, Valencia, CA, USA) and DNase treated (RNase-free DNase Set (Qiagen) or DNase I (Invitrogen)). The RNA concentrations were measured with NanoDrop ND-1000 (Thermo Fisher Scientific, Waltham, MA, USA) and RNA quality was controlled by Experion analysis (Bio-Rad Laboratories, Hercules, CA, USA). All subsequent steps of the microarray analysis were carried out at the Finnish DNAMicroarray Centre utilising the Sentrix Human Illumina 6 V2 Expression BeadChips (Illumina, San Diego, CA, USA), which contains over 47000 known genes, gene candidates, and splice variants. RNA sample ( $300 \mathrm{ng}$ each) was used as template for producing double-stranded cDNA, and then biotinylated cRNA using the Illumina RNA TotalPrep Amplification Kit (Ambion Inc., Austin, TX, USA). The labelled cRNA was purified and hybridised to the BeadChip at $55^{\circ} \mathrm{C}$ for $16 \mathrm{~h}$ following the Illumina WholeGenome Gene Expression Protocol for BeadStation. Hybridisation was detected with Cyanine3-streptavidine (GE Healthcare, Little Chalfont, UK) and the arrays were scanned with the Illumina BeadArray Reader. Normalisation and statistical analyses of the microarray data were performed using the statistical software $\mathrm{R}$ package limma (http://www.R-project.org) or Sigma Stat 3.1 (SPSS Inc., Chicago, IL, USA). The expression levels were analysed using 
Table 2 Serum HE4 and CAI 25 concentrations in patients with endometriosis, endometrial, and ovarian cancer

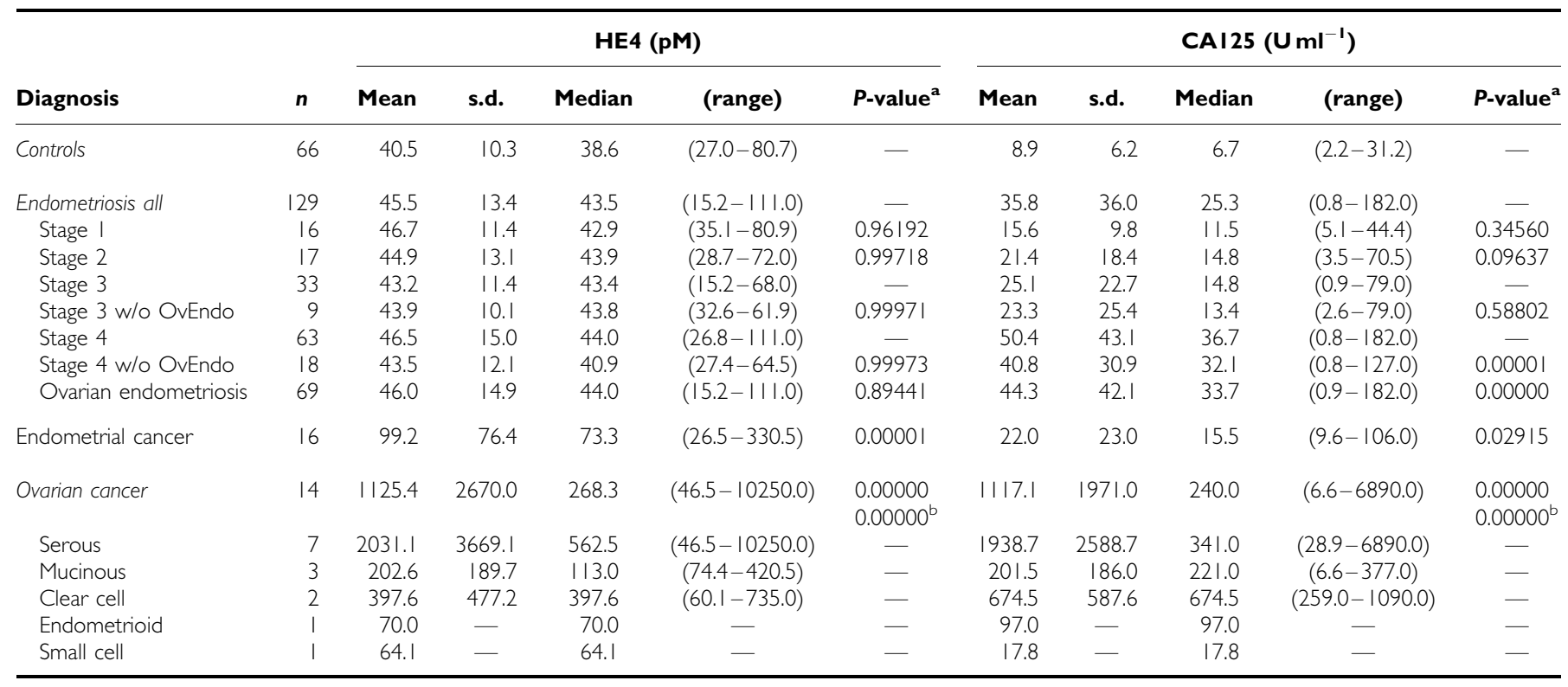

${ }^{a}$ Tukey's multiple comparisons of means, in comparison to the healthy controls. ${ }^{b}$ Comparison to patients with ovarian endometriosis.

the probes ILMN_1706612 and ILMN_1799120 for WFDC2 and ILMN_1736316 for MUC16.

\section{Statistical analyses}

The statistical analyses of serum HE4 and CA125 concentrations alone and in combination were performed using Tukey's multiple comparisons of means with $95 \%$ family-wise confidence level.

The classification capability of the HE4 and CA125 markers, alone and together, was assessed using the binary and multinomial logistic regression models with leave-one-out cross-validation. In the cross-validation each sample in turn was reserved for testing whereas the others were used in the model building. The sensitivity at $95 \%$ specificity and the proportion of correctly classified samples (accuracy) was calculated for each cross-validated regression model. Also, for each binary model, the receiver operator characteristic curves were constructed and the area under the curve was used to summarise the overall performance of the regression model.

\section{RESULTS}

\section{Serum HE4 and CA125 concentrations}

The mean serum HE4 concentrations were similar and below the 70 pM limit for elevated value (Moore et al, 2008a) in patients with endometriosis (mean $45.5 \mathrm{pM}$ ) and in healthy controls (40.5 pM), irrespective of the disease classification or the presence of ovarian endometrioma (ASRM stage 1: $46.7 \mathrm{pM}$; stage 2: $44.9 \mathrm{pM}$; stage 3: 43.2 pM; stage 4: 46.5 pM; OvEndo: 46.0 pM). However, in addition to the highly increased HE4 concentration in patients with ovarian cancer (1125.4 pM), the HE4 serum concentration was significantly elevated also in patients with endometrial cancer $(99.2 \mathrm{pM}$, $P<0.001)$. The levels of HE4 in different types of ovarian cancer were highest in serous (2031.1 pM, $n=7)$ carcinomas, whereas it was clearly elevated also in clear-cell $(397.6 \mathrm{pM}, n=2)$ and mucinous $(202.6 \mathrm{pM}, n=3)$ carcinomas. The serum concentrations in the different patient groups are presented in Table 2 and Figure 1.

The serum levels of CA125 were highest in patients with ovarian cancer (mean $1117.1 \mathrm{U} \mathrm{ml}^{-1}, P<0.001$ ) but were also significantly

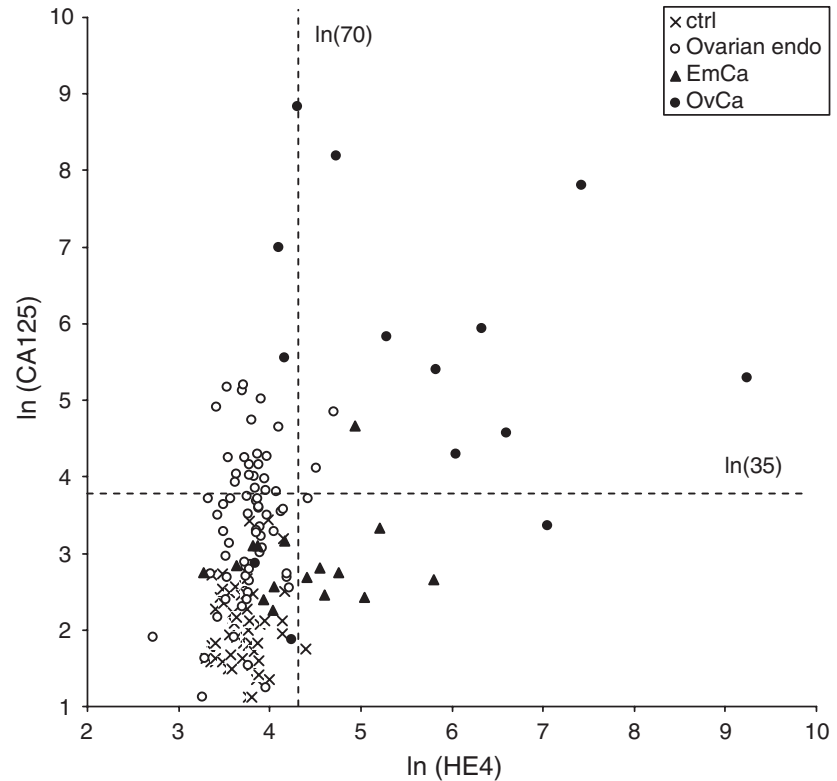

Figure I Correlation between serum HE4 (pM) and CAI25 ( $\left.\mathrm{Uml}^{-1}\right)$ concentrations as In-transformation in patients with ovarian endometriosis (Ovarian endo, $\mathrm{O}$ ), ovarian cancer (OvCa, O), endometrial cancer $(E m C a, \mathbf{\Delta})$, and controls (ctrl, X). The broken lines show the threshold values for positive diagnosis of CAI25 $\left(35 \mathrm{Uml}^{-1}\right)$ and HE4 (70 pM, according to Moore et al (2008a)).

$(P<0.001)$ elevated in patients with ovarian endometrioma $\left(44.3 \mathrm{U} \mathrm{ml}^{-1}\right)$ and advanced non-ovarian endometriosis (ASRM stage $\left.4,40.8 \mathrm{U} \mathrm{ml}^{-1}\right)$ as compared with healthy controls $\left(8.9 \mathrm{U} \mathrm{ml}^{-1}\right)$. These concentrations were also higher than the threshold value for elevated CA1 25 result $\left(35 \mathrm{U} \mathrm{ml}^{-1}\right)$. The concentration increased with increasing ASRM stage of endometriosis (Table 2). However, the median concentration $\left(33.7 \mathrm{U} \mathrm{ml}^{-1}\right)$ in patients with endometrioma is below the threshold value for elevated CA125 result. In sera of patients with endometrial cancer the level of CA125 $\left(22.0 \mathrm{U} \mathrm{ml}^{-1}\right)$ was also significantly $(P=0.029)$ higher than in healthy controls even though clearly lower than the threshold value. 
Sensitivities for the separation of the patient groups by HE4, CA125, or their combination were calculated for each two-wise comparison at specificity of $95 \%$. To differentiate the patients with ovarian cancer from healthy controls, the combination of CA125 and $\mathrm{HE} 4$ relative to $\mathrm{CA} 125$ or $\mathrm{HE} 4$ alone resulted in the highest accuracy (96.3\%) and sensitivity (92.9\%; Table 3). Furthermore, the combination had the highest accuracy (94.0\%) and sensitivity (78.6\%) also for differential diagnosis of patients with ovarian cancer from those with ovarian endometriosis. The combination also differentiates ovarian endometriosis from healthy controls almost as accurately as CA125 alone, even though HE4 alone is a poor marker for endometriosis. Finally, the combination of HE4 and CA125 had the highest accuracy $(81.9 \%)$ also in the three-wise comparison between the ovarian cancer, ovarian endometriosis, and healthy controls.

\section{Expression of HE4 and CA125 encoding genes}

The mRNA expression of WFDC2 (encoding HE4) and MUC16 (encoding CA125) in the tissue samples is shown in Table 4. The expression in ovarian cancer was compared to that of ovarian endometrioma, and the expression in endometrial cancer and nonovarian endometriotic lesions was compared with that of the normal endometrium of healthy controls. The expression of WFDC2 was significantly $(P<0.05)$ higher in ovarian cancer (median of $\log 2$ intensity value 9.25) than in the ovarian

Table 3 Tumour marker accuracy and sensitivity at 95\% specificity for ovarian cancer and ovarian endometriosis

\begin{tabular}{lccc}
\hline Markers & Accuracy (\%) & ROC-AUC (\%) & Sensitivity (\%) \\
\hline OrCa vs OvEndo & & & \\
CAI25+HE4 & 94.0 & 91.3 & 78.6 \\
CAI25 & 92.8 & 77.0 & 64.3 \\
HE4 & 91.6 & 91.9 & 71.4 \\
OrCa vs ctrl & & & \\
CAI25+HE4 & 96.3 & 91.1 & 92.9 \\
CAI25 & 96.3 & 91.7 & 78.6 \\
HE4 & 93.8 & 95.5 & 78.6 \\
OvEndo vs ctrl & & & \\
CAI25+HE4 & 82.2 & 86.8 & 62.3 \\
CAI25 & 83.0 & 87.7 & 60.9 \\
HE4 & 60.7 & 60.5 & \\
OvCa vs OvEndo vs ctrl & & & \\
CAI25+HE4 & 81.9 & & \\
CAI25 & 81.2 & & \\
HE4 & 59.7 & & \\
\hline
\end{tabular}

OvCa $=$ ovarian cancer, OvEndo $=$ ovarian endometriosis. endometrioma (6.73) with fold change (FC) of 5.7. However, the 1.9-fold higher expression in endometrial cancer (8.61) did not reach significance when compared with healthy endometrium (7.67). The expression data is, thus, in line with the differential serum concentrations observed for HE4 in the different patient groups and controls. In contrast, the mRNA expression of MUC16 was relatively stable in the various specimens, whereas the FC between all comparisons was between 0.7 and 1.3.

\section{DISCUSSION}

HE4 is a novel serological marker used especially for ovarian cancer diagnosis (Hellström et al, 2003; Gagnon and Ye, 2008; Hellström and Hellström, 2008; Moore et al, 2008a). Because of its high sensitivity, it is useful also for detecting stage I ovarian cancer (Havrilesky et al, 2008; Moore et al, 2008a). Furthermore, HE4 has been suggested as a biomarker for the diagnosis of endometrial cancer (Moore et al, 2008b). Currently, several biomarker panels are being evaluated to increase the sensitivity and specificity of ovarian cancer diagnosis. The combination of CA125 and HE4 with, or without, other biomarkers such as Glycodelin, Plau-R, MUC-1, PAI-1 (Havrilesky et al, 2008), SMRP (Hellström and Hellström, 2008; Moore et al, 2008a), CA72-4, and osteopontin (Moore et al, 2008a) has been evaluated to improve ovarian cancer diagnosis. The data suggest that by combining these markers the predictive accuracy in ovarian malignancy is better than by applying any of the markers alone. The panel of biomarkers including HE4 has been evaluated also for monitoring the recurrence of ovarian cancer (Havrilesky et al, 2008; Moore et al, 2009).

In female tissues, HE4 immunoreactivity has been shown to be highest in glandular epithelium of the genital tract, including endocervical glands, endometrial glands, fallopian tubes, and Bartholin's glands (Drapkin et al, 2005; Galgano et al, 2006). In contrast to the normal ovarian surface epithelium, which does not express HE4, cortical inclusion cysts lined by metaplastic Müllerian epithelium have been shown to express the protein abundantly (Drapkin et al, 2005). The expression of HE4 protein in ovarian tumours is highest in serous carcinomas but immunostaining has been detected also in the vast majority of ovarian endometrioid and clear-cell carcinomas (Drapkin et al, 2005; Galgano et al, 2006). In addition to ovarian carcinoma, some pulmonary, endometrial, and breast adenocarcinomas have been shown to express HE4 (Galgano et al, 2006). Although the protein has been detected in both normal and malignant endometrium, the expression of HE4 in the endometriotic lesions is only superficially known. Recently, Moore et al (2008a) analysed HE4 and eight other biomarkers in the sera of 166 patients with ovarian cancer or with several other kinds of pelvic masses, of whom 29 had endometriosis. They showed that the HE4 and CA125 concentrations were the best combination of biomarkers to distinguish

Table 4 Expression of WFDC2 (HE4) and MUCI6 (CAI25) mRNA in tissue specimens of endometrium, non-ovarian and ovarian endometriosis, endometrial cancer, and ovarian cancer as $\log 2$ intensity values

\begin{tabular}{|c|c|c|c|c|c|c|c|c|c|c|c|}
\hline Sample group & $\mathbf{N}$ & \multicolumn{5}{|c|}{ WFDC2 } & \multicolumn{5}{|c|}{ MUCI6 } \\
\hline $\mathrm{E}$ of endo patient & 64 & 7.75 & 7.17 & 8.28 & $1.1^{\mathrm{b}}$ & $N S^{b}$ & 7.21 & 6.86 & 7.63 & $1.1^{\mathrm{b}}$ & $N S^{b}$ \\
\hline Non-ovarian endo & 149 & 7.03 & 6.65 & 7.44 & $0.6^{\mathrm{b}}$ & $<0.05^{\mathrm{b}}$ & 6.62 & 6.48 & 6.84 & $0.7^{\mathrm{b}}$ & $<0.05^{b}$ \\
\hline Ovarian endo & 28 & 6.73 & 6.45 & 7.13 & & & 6.49 & 6.37 & 6.65 & & \\
\hline $\mathrm{EmCa}$ & 14 & 8.61 & 8.06 & 9.24 & $1.9^{\mathrm{b}}$ & NS & 6.28 & 6.14 & 7.29 & $0.6^{b}$ & $<0.05^{b}$ \\
\hline
\end{tabular}

$\mathrm{E}=$ endometrium; EmCA = endometrial cancer; endo = endometriosis; OvCa = ovarian cancer; NS = not significant. ${ }^{\mathrm{a}}$ Dunn's method. ${ }^{\mathrm{b}} \mathrm{Compared}$ to endometrium specimens of healthy controls. ${ }^{\mathrm{C}}$ Compared to ovarian endometriomas. 
ovarian cancer patients from those with other pelvic masses. However, the types of endometriosis lesions in these patients were not described.

In agreement with other recent studies (Hellström et al, 2003; Gagnon and Ye, 2008; Hellström and Hellström, 2008; Moore et al, 2008a, b), we detected increased HE4 concentration in patients with ovarian and endometrial cancer. The present data demonstrate that neither the expression of HE4 encoding gene in the endometriotic lesions nor serum HE4 concentration in the patients with endometriosis with any types of endometriosis is increased. It is of specific interest to note that HE4 is not increased even in patients with ovarian endometriosis. In contrast, the serum level of CA125 was increased in patients with advanced endometriosis and ovarian endometriomas, as expected. It should be noted that endometriosis is typically diagnosed at young adult age (25-35 years) and often disappears after menopause, whereas the incidence of ovarian cancer increases in older women (highest incidence at the age of 50-60 years). Interestingly, it has been reported that the concentration of HE4 increases with age in healthy postmenopausal woman whereas CA125 does not (Lowe et al, 2008).

Thus, measuring both HE4 and CA125 together, rather than either of them alone, provides a more accurate tool for differential diagnosis of patients with ovarian cancer and ovarian endometriotic cysts from healthy subjects. It may also help clinicians in the follow-up of patients suffering from advanced endometriosis when considering the possibility of malignant transformation of the lesions. Within the patients with ultrasound-detected ovarian mass, the high serum HE4 with high CA125 would suggest the presence of ovarian cancer whereas elevated CA125 without elevated HE4 would direct towards advanced or ovarian endometrioma or other benign conditions. Furthermore, the

\section{REFERENCES}

(1997) Revised American Society for Reproductive Medicine classification of endometriosis: 1996. Fertil Steril 67: 817-821

Benedet JL, Bender H, Jones 3rd H, Ngan HY, Pecorelli S (2000) FIGO staging classifications and clinical practice guidelines in the management of gynecologic cancers, FIGO Committee on Gynecologic Oncology. Int $J$ Gynaecol Obstet 70: 209-262

Drapkin R, von Horsten H, Lin Y, Mok S, Crum C, Welch W, Hecht J (2005) Human epididymis protein 4 (HE4) is a secreted glycoprotein that is overexpressed by serous and endometrioid ovarian carcinomas. Cancer Res 65: $2162-2169$

Gagnon A, Ye B (2008) Discovery and application of protein biomarkers for ovarian cancer. Curr Opin Obstet Gynecol 20: 9-13

Galgano M, Hampton G, Frierson HJ (2006) Comprehensive analysis of HE4 expression in normal and malignant human tissues. Mod Pathol 19: $847-853$

Giudice L, Kao L (2004) Endometriosis. Lancet 364: 1789- 1799

Havrilesky L, Whitehead C, Rubatt J, Cheek R, Groelke J, He Q, Malinowski D, Fischer T, Berchuck A (2008) Evaluation of biomarker panels for early stage ovarian cancer detection and monitoring for disease recurrence. Gynecol Oncol 110: 374-382

Hellström I, Hellström K (2008) SMRP and HE4 as biomarkers for ovarian carcinoma when used alone and in combination with CA125 and/or each other. Adv Exp Med Biol 622: 15-21

Hellström I, Raycraft J, Hayden-Ledbetter M, Ledbetter J, Schummer M, McIntosh M, Drescher C, Urban N, Hellström K (2003) The HE4 (WFDC2) protein is a biomarker for ovarian carcinoma. Cancer Res 63: $3695-3700$

Kitawaki J, Ishihara H, Koshiba H, Kiyomizu M, Teramoto M, Kitaoka Y, Honjo H (2005) Usefulness and limits of CA-125 in diagnosis of endometriosis without associated ovarian endometriomas. Hum Reprod 20: $1999-2003$

Lowe KA, Shah C, Wallace E, Anderson G, Paley P, McIntosh M, Andersen MR, Scholler N, Bergan L, Thorpe J, Urban N, Drescher CW (2008) Effects of personal characteristics on serum CA125, mesothelin, and HE4 levels in healthy postmenopausal women at high-risk for ovarian cancer. Cancer Epidemiol Biomarkers Prev 17: 2480-2487 elevated serum HE4 concentration with normal CA125 concentration would suggest the presence of either ovarian or possibly other type of cancer, for example endometrial cancer.

The greatest benefit of highly specific differentiation between ovarian cancer and endometriosis may well be found in identification of ovarian cancer in the early non-symptomatic stage. A high proportion of ovarian cancers are diagnosed at an advanced stage with a dismal survival rate. In contrast, the 5-year survival rate for stage I disease with the malignancy confined to the ovary is above $90 \%$. This emphasises the importance of detecting the ovarian cancers at their early stage to improve the mortality rate.

In summary, the serum concentration of HE4, a novel biomarker for ovarian cancer, was not increased in patients with ovarian endometrioma or any other types of endometriosis, whereas the serum CA125 concentration was increased in patients with advanced endometriosis. The results, thus, suggest that the serum HE4 concentration is a valuable marker to better distinguishing patients with ovarian malignancies from those suffering from the benign ovarian endometriotic cysts.

\section{ACKNOWLEDGEMENTS}

This study was supported by Finnish Funding Agency for Technology and Innovation (projects 40343/05 and 599/05); Biotop Oy, Finland; Hormos Medical Ltd, Finland (subsidiary of QuatRx Pharmaceutical, USA); Genolyze Oy, Finland; The Finnish Medical Foundation (AA, JHu); Drug Discovery Graduate School (KH); and Graduate School in Computational Biology, Bioinformatics, and Biometry (JHi).

Markman M (1997) The role of CA-125 in the management of ovarian cancer. Oncologist 2: 6-9

Mol B, Bayram N, Lijmer J, Wiegerinck M, Bongers M, van der Veen F, Bossuyt P (1998) The performance of CA-125 measurement in the detection of endometriosis: a meta-analysis. Fertil Steril 70: 1101-1108

Moore R, Brown A, Miller M, Badgwell D, Lu Z, Allard W, Granai C, Bast $\mathrm{RJ}$, Lu K (2008b) Utility of a novel serum tumor biomarker HE4 in patients with endometrioid adenocarcinoma of the uterus. Gynecol Oncol 110: $196-201$

Moore R, Brown A, Miller M, Skates S, Allard W, Verch T, Steinhoff M, Messerlian G, DiSilvestro P, Granai C, Bast RJ (2008a) The use of multiple novel tumor biomarkers for the detection of ovarian carcinoma in patients with a pelvic mass. Gynecol Oncol 108: $402-408$

Moore R, McMeekin D, Brown A, DiSilvestro P, Miller M, Allard W, Gajewski W, Kurman R, Bast RJ, Skates S (2009) A novel multiple marker bioassay utilizing HE4 and CA125 for the prediction of ovarian cancer in patients with a pelvic mass. Gynecol Oncol 112: 40-46

Nagle C, Olsen C, Webb P, Jordan S, Whiteman D, Green A (2008) Endometrioid and clear cell ovarian cancers: a comparative analysis of risk factors. Eur J Cancer 44: 2477-2484

Ness R (2003) Endometriosis and ovarian cancer: thoughts on shared pathophysiology. Am J Obstet Gynecol 189: 280-294

Nisolle M, Donnez J (1997) Peritoneal endometriosis, ovarian endometriosis, and adenomyotic nodules of the rectovaginal septum are three different entities. Fertil Steril 68: 585-596

Sato N, Tsunoda H, Nishida M, Morishita Y, Takimoto Y, Kubo T, Noguchi M (2000) Loss of heterozygosity on 10q23.3 and mutation of the tumor suppressor gene PTEN in benign endometrial cyst of the ovary: possible sequence progression from benign endometrial cyst to endometrioid carcinoma and clear cell carcinoma of the ovary. Cancer Res 60: 7052-7056 Seeber B, Sammel M, Fan X, Gerton G, Shaunik A, Chittams J, Barnhart K (2008) Panel of markers can accurately predict endometriosis in a subset of patients. Fertil Steril 89: $1073-1081$

Terry K, Sluss P, Skates S, Mok S, Ye B, Vitonis A, Cramer D (2004) Blood and urine markers for ovarian cancer: a comprehensive review. Dis Markers 20: $53-70$ 